\title{
Anforderungen an ein Daten-Backend-System zur Unterstützung industrieller Datenanalyse-Anwendungen in digitalen Engineering-Prozessen dynamischer Wertschöpfungsnetzwerke
}

\section{Requirements for a data backend system to support industrial data analysis applications in digital engineering processes of dynamic value networks}

\author{
Andreas Eiden ${ }^{1 *}$, Jonas Gries ${ }^{1}$, Thomas Eickhoff ${ }^{1}$, Jens C. Göbel ${ }^{1}$ \\ ${ }^{1}$ Institute of Virtual Product Engineering, University of Kaiserslautern \\ ${ }^{*}$ Korrespondierender Autor: \\ Andreas Eiden \\ TU Kaiserslautern \\ Lehrstuhl für Virtuelle Produktentwicklung \\ Gottlieb-Daimler-Str. 44 \\ 67663 Kaiserslautern \\ Telefon: 00496312053990 \\ Mail: eiden@mv.uni-kl.de
}

\begin{abstract}
Industrial data analytics needs well-structured and linked data from different data sources. The increasing mass of data, scattered IT-structures and a lack of knowledge, especially in small and medium-sized companies (SMEs) are factors that hinder the usage of data analytics. The goal of the research project AKKORD is to build a toolkit for companies to facilitate distributed and integrated industrial data analytics inside valueadding networks. A core part of this toolkit is a data backend system, which collects and links data from different source systems together in a single meta-model. This paper describes the requirements analysis of the data backend system by conducting structured interviews and workshops.
\end{abstract}

\section{Keywords}

Digital Engineering Processes, PLM, Product Data Integration; Interoperability in Product Design; Digital Product Twin 


\section{Motivation}

Die industrielle Datenanalyse im Produktlebenszyklus ist heute ein zentrales Instrument, um Wissen über Produkte und digitale Engineeringprozesse, welche überwiegend von Ingenieuren durchgeführt werden, zu generieren. Hierbei stehen Produkte und Prozesse in einem engen Zusammenhang, da die Planung, Definition und Dokumentation eben jener Produkte das Ziel der durch IT-Technologie unterstützten digitalen Engineeringprozesse ist. Die Konzeption von digitalen Zwillingen [1] bietet für Firmen umfassende Möglichkeiten zur Datenanalyse, beispielsweise im Kontext von Prozessoptimierung [2] oder einer effizienteren Produktion [3]. Die dadurch immer komplexer werdenden IT-Strukturen erfordern eine Durchgängigkeit von Daten über sämtliche Bereiche des Produktlebenszyklus [4, 5], sowie über Firmengrenzen hinweg. Die steigende Komplexität der Produkte sorgt für eine Verlagerung von Kompetenzen von einzelnen Firmen hin zu Werschöpfungsnetzwerken, welche aufgrund der vielfältigen Zusammenarbeit über den ganzen Produktlebenzyklus hinweg einen dynamischen Charakter haben [6]. Darüber hinaus ist auch das Monetarisieren von Daten in diesen Wertschöpfungsnetzwerken als neues Geschäftsmodell möglich, sofern eine ausreichende Infrastruktur hierfür vorhanden ist. Problematisch ist in diesem Fall, dass Daten als immaterielles Gut schwierig zu kontrollieren sind, da sie nach dem Erwerb nicht verbraucht werden, sondern dem Käufer weiter als Kopie zur Verfügung stehen könnten [7].Trotz der bestehenden (Teil)Lösungen für diese Probleme schaffen viele Unternehmen, insbesondere kleine und mittelständische Unternehmen (KMU), es nicht, die hiermit verbundenen Potenziale mit eigenen Mitteln zu erschließen [8].

Ein großes Problem hierbei ist die Anzahl der gleichzeitig auftretenden Neuerungen. Datenstrukturen müssen überarbeitet, Datenanalysestrategien durchdacht, entsprechende Software genutzt und die Mitarbeiter in all diesen Punkten geschult werden. Zur Verbesserung dieser Situation wird im Forschungsprojekt AKKORD - Vernetzte und integrierte Anwendung industrieller Datenanalyse für die wertschaffende, kompetenzorientierte Kollaboration in dynamischen Wertschöpfungsnetzwerken [9] - ein Referenzbaukasten entwickelt, welcher besonders für KMU die Einführung von Datenanalyse unterstützt. Der Referenzbaukasten besteht aus vier Leistungsbereichen, welche Lösungen für die Analyse von Daten mithilfe vorgefertigter Module, das Erarbeiten neuer Kollaborations- und Geschäftsmodelle über Unternehmensgrenzen hinaus, den gezielten Kompetenzaufbau der Arbeitnehmer, sowie die Vernetzung und Bereitstellung von Daten beinhalten. Der im Fokus stehende Leistungsbereich Daten-Backend-System soll verschiedene heterogene Datenquellen integrieren und somit Datenanalysen aus diesem vernetzten Backend heraus ermöglichen.

\section{Stand der Technik}

Datenvernetzung ist ein bereits lange diskutiertes Themenfeld in Forschung und Industrie. Durch die steigende Anwendung des Model-Based Systems Engineering (MBSE) können durchgängige Produktmodelle erstellt werden, welche ausgehend von Anforderungen bis hin zur interdisziplinären Modellierung des Produktes bzw. seiner Produktionsanlage eine Rückverfolgbarkeit herstellen. Eine Verwaltung dieser Modelle kann durch Product Lifecycle Management (PLM)-Systeme vorgenommen werden [10, 11], was sich auch im Ansatz des System Lifecycle Management (SysLM) ausdrückt $[12,13]$. Die in diesen Ansätzen realisierte Vernetzung endet allerdings mit dem Übergang der logischen Systemarchitektur in die disziplinspezifische Modellierung. Die ab dieser Stelle erzeugten Modelle haben, häufig durch die Verwendung unterschiedlicher Verwaltungssysteme, keine maschinenlesbare Verbindung mehr zu den vorherigen Phasen des Lebenszyklus. Woll et al [14] beschreiben eine Möglichkeit, die Entwicklungsartefakte des Model-Based Systems Engineering mithilfe einer ontologiebasierten Datenintegration miteinander zu verbinden. 
Die Entwicklung von smarten Produkten [15, 16] führt aufgrund der hohen Komplexität in der Produktentwicklung zu einem steigenden Bedarf an besseren Methoden und Tools, insbesondere auch zu besser vernetzten Informationen über Produkte und Prozesse [17].

Ein Ansatz, der diese Lücke schließen soll, ist das sogenannte Closed-Loop Systems Engineering. Hierfür werden die MBSE-Modelle aus der frühen Lebenszyklusphase mit Daten aus virtuellen Prototypen und später den digitalen Zwillingen der realen Instanz verknüpft, um diese Daten in der Produktplanung und -entwicklung nachfolgender Generationen einzusetzen und diese iterativ zu verbessern. [18]

Neben Ansätzen aus dem Model-Based Systems Engineering können die Lösungsalternativen in Vernetzungsmöglichkeiten mit oder ohne eine Integrationsplattform geclustert werden. Grundlegend für viele Vernetzungsmöglichkeiten sind serviceorientierte Architekturen [19, 20] und Standards wie die ISO 10303 [21, 22]. Interoperabilität durch die Nutzung von Webstrukturen wird u.a. in [23] und [24] beschrieben. Das Projekt Open Services for Lifecycle Collaboration (OSLC) versucht einen gemeinsamen Standard für eine Datenintegration durch verlinkte Daten mithilfe von Semantic Web-Technologien zu schaffen. Aufbauend auf einem gemeinsamen Minimalstandard werden Anwendungsprofile betrachtet, welche ein vereinheitlichtes Vokabular besitzen und anwendungsspezifische Integrationsszenarien, z.B. das Änderungs- oder Anforderungsmanagement, beschreiben [25]. Einen ähnlichen Ansatz im Bereich der Produktion mithilfe von OPC-UA beschreibt [26].

Basierend auf den beschriebenen grundlegenden Techniken und Konzepten gibt es eine Reihe von Integrationsplattformen wie in [27], [28], [29] und [30]. Diese werden im Allgemeinen speziell für unterschiedliche Use-Cases entworfen und integrieren die in diesem fachlichen Kontext notwendigen Daten. In diesem Bereich gibt es auch eine Reihe kommerzieller Lösungen, wie Intercax Syndeia [31] oder eQube BI [32].

Um den Bedarf an vernetzten Daten über den gesamten Lebenszyklus hinweg, welcher für Datenanalysen benötigt wird, zu decken, braucht es einen über das MBSE oder Integrationsplattformen für einzelne Use-Cases hinausgehenden Ansatz, welcher explizit auch die Verteilung, Autonomie und Heterogenität aller notwendigen Daten berücksichtigt [33]. Ein möglicher Lösungsansatz stellt eine Plattform dar, auf welcher ohne spezifische Anwendungsfälle die Metadaten aus verschiedenen Datenbanken und IT-Systemen entlang des Lebenszyklus verlinkt werden [34]. Die in Abbildung 1 dargestellte Integrationsplattform verwendet einen leichtgewichtigen Kern, um heterogene IT-Systeme mithilfe von spezifischen Wrappern zu verknüpfen.

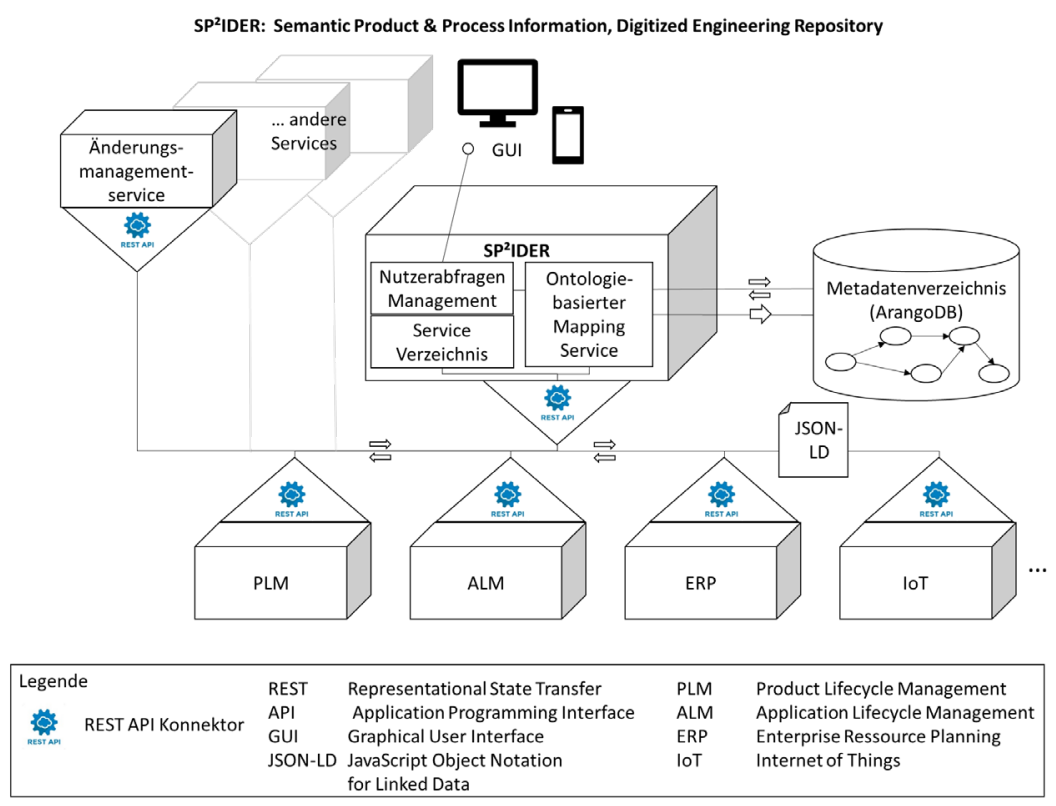

Bild 1: Beispiel einer Integrationsplattform aus [34] 


\section{Forschungsproblem und Forschungsziel}

Zur Durchführung von integrierten und wertschöpfungsübergreifenden Datenanalysen in KMU ist es notwendig, dass die Daten bereits in einer möglichst aufbereiteten Form vorhanden sind, über Unternehmensgrenzen hinweg freigegeben werden können und als Basis neuer Geschäftsmodelle dienen können. Insbesondere kleinere Unternehmen stehen hier vor den Herausforderungen kleiner Budgets, nur wenig Fachkompetenz im eigenen Unternehmen und fehlender Tools, um diese Probleme zu lösen. Während einzelne Aspekte dieser Problemstellung bereits mit den Methoden und Tools, welche in Kapitel 2 vorgestellt werden, gelöst werden können, bedarf es für KMU einer einfachen Lösung, welche ein Gesamtkonzept darstellt. Das Projekt AKKORD versucht deshalb, einen integrierten Referenzbaukasten zu schaffen, welcher KMU eine Gesamtlösung für all diese Aufgaben anbietet. Als erster Schritt soll dieser Referenzbaukasten mit den Projektpartnern erarbeitet und getestet werden. Speziell für den Leistungsbereich des Daten-Backend-Systems ergeben sich folgende Forschungsfragen:

- Welche Anforderungen ergeben sich an ein Daten-Backend-System aus Sicht eines $\mathrm{KMU}$, das industrielle Datenanalyse damit betreiben will?

- Welche Anforderungen ergeben sich an ein Daten-Backend-System aus Sicht eines Lösungsanbieters des integrierten Referenzbaukastens oder eines seiner Leistungsbereiche?

- Inwieweit können bereits existierende Lösungsansätze zur Datenvernetzung auch im angedachten Referenzbaukasten zur integrierten Anwendung industrieller Datenanalyse genutzt werden, um die Bedürfnisse von KMU zu erfüllen?

Die erste Forschungsfrage wird im Kontext des Projekts mit den prototypischen Anwendungspartnern adressiert. Die vier Partner stehen mit ihren spezifischen Problemen für eine Implementierung bereit, aus der heraus sich dann durch eine Verallgemeinerung eine Lösung im Sinne des Referenzbaukastens ergibt.

Zur Betrachtung der zweiten Forschungsfrage tragen die sechs Unternehmen bei, die in diesem Projekt als Lösungsanbieter fungieren und die mit ihren spezifischen Fähigkeiten und Leistungen bereits mögliche Teillösungen anbieten, welche auch Teil des später aufzubauenden Referenzbaukastens werden sollen.

Das Ziel hinter den zu beantwortenden Forschungsfragen ist die Erarbeitung eines Lastenund Pflichtenheftes, welches für den Aufbau eines Daten-Backend-Systems genutzt werden kann, um diesen im Referenzbaukasten des AKKORD-Projekts einzubinden. Daneben können die Ergebnisse auch genutzt werden, um einen Beitrag zur durchgängigen Vernetzung von Daten und Prozessen über den Produktlebenszyklus hinweg zu leisten und neben der Datenanalyse auch weitere Tätigkeiten von Ingenieuren zu erleichtern.

\section{Strukturiertes Interview zur qualitativen Erhebung der Anforderungen}

Um das Ziel des integrierten Lasten- und Pflichtenheftes zu erreichen, wurde als Methodik eine Kombination aus Recherchen,strukturierten Interviews [35] und Workshops gewählt. In einer initialen Recherche wurden mögliche Lösungsansätze auf ihre Eignung und Struktur hin untersucht. Die wichtigsten Ergebnisse der initialen Recherche sind in Kapitel 2 dargestellt. Die übergreifenden Gemeinsamkeiten der verschiedenen Ansätze und IT-Tools wurden in die Kategorien Daten, Datenquellsysteme, Nutzerinterface, Prozessunterstützung, Services, Rollen \& Rechte sowie Safety \& Security geclustert, aus welchen Interviewfragen erarbeitet wurden.

Um das Ziel einer umfassenden Lösung über einzelne Disziplinen oder Engineeringprozesse bzw. Lebenszyklusbereiche hinweg zu adressieren, wurden die 
Interviewpartner mit einer großen Bandbreite ausgewählt. Interviews mit den im Folgenden beschriebenen Fragen wurden mit Mitarbeitern der verschiedenen Anwendungspartnern des Projekts aus den Bereichen Konstruktion, Industrial Engineering und Qualitätsmanagement durchgeführt. Für die Lösungsanbieter und Entwicklungspartner waren u.a. Datenanalysten und Projektmanager am Interview beteiligt.

Der erste Fragebereich „Daten“ befasst sich mit den Daten an sich. Die Verfügbarkeit von Daten ist eine essenzielle Grundlage für eine Datenanalyse. Aus diesem Grund muss mit den folgenden Fragen geklärt werden, welche konkreten Daten und beschreibenden Metadaten in den Anwendungsfällen benötigt werden und in welchen Formaten diese vorliegen:

- Welche Daten existieren im Unternehmen entlang des Produktlebenszyklus?

- Welche Formate haben diese Daten?

- Welche Metadaten existieren zu diesen Daten?

Im zweiten Bereich „Datenquellsysteme“ werden die zuvor diskutierten Daten den im Unternehmen vorliegenden Datenquellsystemen zugeordnet. Aufgrund der stark unterschiedlichen Anwendungsfälle und IT-Strukturen in KMU wurde versucht, mit den Anwendungspartnern im Projekt AKKORD ein möglichst heterogenes Spektrum an Firmen abzudecken, um eine Übertragbarkeit auf weitere Unternehmen sicherzustellen. Aus diesen Punkten heraus ergeben sich folgende Fragen:

- Welche Datenquellsysteme werden benötigt, um Daten für den gewünschten Anwendungsfall zur Verfügung zu stellen?

- Welche Datenquellsysteme existieren zusätzlich im Unternehmen, die über den Anwendungsfall hinaus weitere Informationen und Daten zur Verfügung stellen können?

Das Nutzerinterface ist die Schnittstelle, mit deren Hilfe der Anwender mit dem DatenBackend-System interagiert. Der Umfang und die Ausgestaltung eines solchen Interfaces variieren von sehr aufwändigen graphischen Dashboards bis hin zu einfachen, textbasierten konsolenartigen Schnittstellen. Um die Anforderungen aus verschiedenen Anwendungsperspektiven aufzunehmen, haben sich folgende Fragen ergeben:

- Wie soll ein Interface für das Daten-Backend-System zur Datenanalyse aussehen?

- Welche Funktionen sollen hier bereitgestellt werden?

- Wie sieht das Nutzungsszenario für die angedachten Use-Cases aus? Wie viele Nutzer arbeiten mit dem System? Ist eine weltweite Nutzung auf Mobilgeräten geplant oder nur On-Premises?

Der Bereich „Prozessunterstützung“ des Interviews widmet sich der Prozesssicht des Daten-Backend-Systems. Die (Engineering-)Prozesse, welche durch eine Datenintegrationslösung unterstützt werden, sind sehr vielfältig, während die Datenanalyse für KMU im Vordergrund steht. Im Projektkontext sind insbesondere die Auswahl der Inputdaten sowie die Vorverarbeitung für Analysen von Interesse. Aus Sicht des Nutzers besteht hier ein starker Zusammenhang mit dem Bereich Nutzerinterfaces, da sich die Prozesse im Interface abbilden lassen müssen bzw. ein flexibel aufgebautes Interface auch die nachträgliche Einbindung weiterer Prozesse unterstützt. Die sich ergebenden Fragen sind die folgenden:

- Wie sieht der Prozess aus, wenn Daten für eine Analyse ausgewählt werden sollen?

- Wie soll das Daten-Backend-System bei der Daten-Vorverarbeitung unterstützen? 
Ergänzend zum Fokusthema Datenanalyse sollen im Bereich „Services“ des Interviews weitere potenzielle Funktionen aus Sicht der Anwender aufgedeckt werden. Die Entwicklung ergänzender Services steht in direkter Verbindung zu dem Bereich Prozessunterstützung sowie Nutzerinterface.

- Welche weiteren Funktionen und Services sind aus Nutzer- bzw. Anbietersicht wünschenswert?

Der Bereich Rollen \& Rechte ist im Projektkontext besonders interessant, da Datenanalysen und dementsprechend auch das Daten-Backend-System unternehmensübergreifend eingesetzt werden sollen. Gleichzeitig ist natürlich auch der immaterielle Charakter der Daten eine Herausforderung, da, sobald diese bekannt sind, das Wissen über Daten nicht mehr rückgängig gemacht werden kann. Dementsprechend ergeben sich folgende Fragen:

- Wer arbeitet in einem Unternehmen mit den verfügbaren Daten?

- Wie soll ein Prozess aussehen, der anderen Mitarbeitern die Möglichkeit eröffnet, mit diesen Daten zu arbeiten?

Als letzter Bereich werden Aspekte der Safety \& Security beleuchtet. Hier gilt es die enorme Heterogenität der Unternehmen und Ihrer Anwendungsfälle zu beleuchten, woraus sich die folgende Frage ergibt:

- Wo liegen die vernetzten Daten? Auf einzelnen Computern, auf einem Server OnPremises, in einer Cloud?

$\mathrm{Da}$ das Daten-Backend-System nicht für sich alleine entstehen soll, sondern im Projektkontext ein Leistungsbereich des zu schaffenden Referenzbaukastens ist, ist auch diese Einbettung ein wichtiges Thema:

- Wie sieht das Zusammenspiel zwischen den verschiedenen Leistungsbereichen des Referenzbaukastens aus?

- Welche Weiterbildungsangebote müssen geschaffen werden, damit Firmen die Leistungsbereiche des Referenzbaukastens nutzen können?

Der obige Fragebogen wurde mit jedem Interviewpartner in ca. 60 Minuten besprochen und mitgeschnitten. Anschließend wurden die Aussagen transkribiert und analysiert. Aus den Interviewmitschnitten und Notizen konnten insgesamt 29 Kernanforderungen extrahiert werden, die teilweise von mehreren Partnern genannt wurden. Zur besseren Strukturierung wurden die Anforderungen in Kategorien eingeteilt. Aus der Summe der Antworten wurden die Kategorien Vernetzung, Metamodell, Infrastruktur, Ausgabe, Services sowie Kompetenzen ermittelt.

Basierend auf den bisherigen Ergebnissen wurde in einem Workshop mit den Forschungspartnern, welche jeweils einen Leistungsbereich des Referenzbaukastens vertreten, ein erster Entwurf des integrierten Lasten- und Pflichtenhefts erstellt. Es beschreibt zum einen die Anforderungen, welche von allen Projektpartnern geäußert wurden, sowie eine Priorisierung der Anforderungen. Als Absicherung der Anforderungen wurden mithilfe von vier Personae während des Workshops die Interaktion eines Geschäftsführers, eines Domänenexperten, eines Datenanalysten und eines Dateningenieurs mit dem 
Referenzbaukasten durchgespielt. Durch die Personae, welche als abstrakte Repräsentation aus einem mittelständischen Unternehmen konzipiert sind, ist sichergestellt, dass mit diesem Lastenheft nicht nur die Bedürfnisse der spezifischen Projektpartner erfüllt werden, sondern es darüber hinaus eine gewisse Allgemeingültigkeit für die Bedürfnisse mittelständischer Unternehmen in den unterschiedlichen Bereichen besitzt. Das hierdurch abgesicherte integrierte Lasten- und Pflichtenheft wurde anschließend von sämtlichen Partnern des Projektes gesichtet, erweitert und in einer finalen Form gemeinsam verabschiedet.

\section{Ergebnisse und Diskussion}

Mithilfe des gewählten methodischen Vorgehens aus Recherchen, strukturierten Interviews und anschließenden Workshops mit mehreren Projektpartnern konnten insgesamt 29 Kernanforderungen an ein Daten-Backend-System abgeleitet werden. Die erhobenen Anforderungen zeigen durch eine Mehrfachnennung in den Interviews insbesondere die wichtigsten Punkte, die bei der Erarbeitung und Implementierung zu beachten sind. Eine Zusammenfassung der wichtigsten genannten Anforderungen wird in den folgenden Unterkapiteln beschrieben.

\subsection{Flexible Datenanbindung}

So wird insbesondere eine möglichst flexible Anbindung bestehender Quellen gefordert. Neben etablierten Verwaltungssystemen wie Enterprise Ressource Planning (ERP), Product Lifecycle Management (PLM) oder Manufacturing Execution System (MES) müssen vor allem auch Hardware-Datensammler, Systeme für die Verwaltung digitaler Zwillinge und loTPlattformen angebunden werden. Hierbei sollen nicht nur einzelne spezifische Systeme koppelbar sein, sondern eine Schnittstellenarchitektur entwickelt werden, mit der flexibel weitere Systeme angebunden werden können. Zur besseren Kompatibilität muss hier soweit wie möglich auf offene Standards wie REST, OSLC und weitere Semantic-Web-Technologie aufgebaut werden. Um ein Daten-Backend-System auch in KMU sinnvoll nutzen zu können, ergibt sich die Anforderung, dass auch Daten, welche nicht in Verwaltungssystemen abgelegt sind, angebunden werden müssen. Als weitgehender De-facto Standard für Unternehmensdaten in KMU haben sich die Officeprogramme von Microsoft etabliert. Stücklisten, Fertigungsdaten, Arbeitspläne und viele weitere Dokumente werden teilweise ausschließlich in diesen Formaten gespeichert.

\subsection{Datenmapping und semantische Aufwertung}

Aufgrund der problematischen Verfügbarkeit und großen Heterogenität der Daten muss das Daten-Backend-System hier nicht nur ein Datenprovider für Datenanalyse sein, sondern mithilfe von Machine Learning-Ansätzen die Integration und Vernetzung der Daten sicherstellen. Um einen Mehrwert an Daten über die reine Verfügbarkeit in einem System hinaus zu schaffen, muss das Daten-Backend-System eine Vernetzung sicherstellen, bei der identische Daten aus verschiedenen Systemen in einer gemeinsamen Struktur abgespeichert werden. Eine semantische Aufwertung der vernetzten Daten wird hierbei als sehr hilfreich eingeschätzt, um relevante Daten zu identifizieren und die Vorverarbeitung so zu beschleunigen. Das Daten-Backend-System muss dabei einen Mittelweg finden, um zwar eine semantische Aufwertung zu schaffen, gleichzeitig aber auch flexibel mit neuen Strukturen umzugehen, ohne jeweils vorher ein komplettes Datenmodell wie z.B. eine Ontologie zu entwerfen. 


\subsection{Weitere Anforderungen}

Um dem hohen Sicherheitsbedürfnis der Unternehmen nachzukommen, soll das DatenBackend-System auch on-Premises, also auf eigenen Servern, genutzt werden können. Während dies für Firmen mit einer bereits bestehenden leistungsstarken Infrastruktur möglich erscheint, muss auch eine Public Cloud Lösung ermöglicht werden, welche die höchsten Ansprüche an die Datensicherheit gewährleistet.

Um gemeinsam mit Partnern an der Auswertung zu arbeiten oder im Rahmen kollaborativer neuer Geschäftsmodelle Daten zu tauschen, müssen Schnittstellen zur Freigabe von Daten vorhanden sein. Eine sehr häufig genannte Anforderung war die Aufwertung des DatenBackend-Systems mit Leitfäden und Assistenzsystemen, welche dem Anwender bei der Nutzung und insbesondere bei der Konfiguration unterstützen. Oft wurde hierbei auch der Wunsch nach einer Unterstützung bei grundlegenden Themen wie z.B. der Datenstrukturierung oder der Datenqualität geäußert.

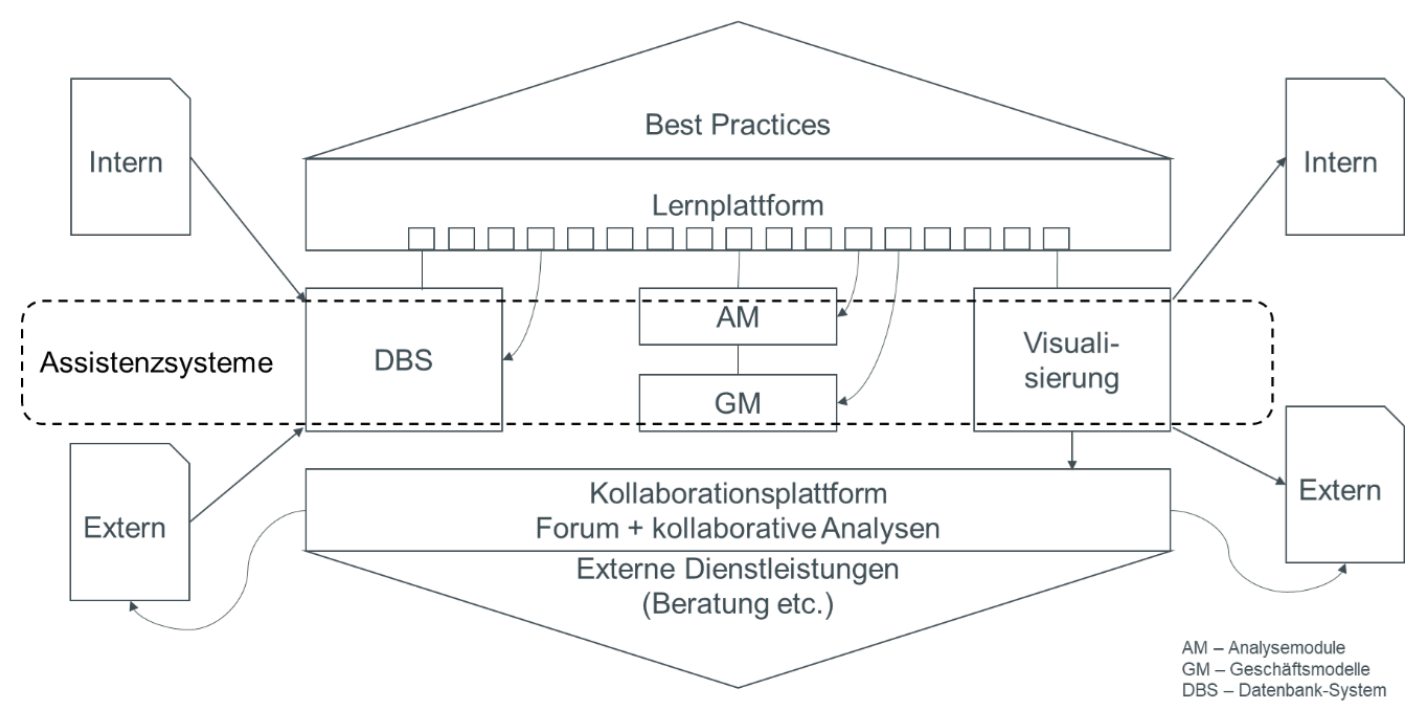

Bild 2: Einbindung des Daten-Backend-Systems (DBS) in den integrierten Referenzbaukasten

Im Rahmen des Forschungsprojekts AKKORD wurde mithilfe von strukturierten Interviews und Workshops ein integriertes Lasten- und Pflichtenheft erstellt, welches den Bedarf der Partnerunternehmen u.a. an ein Daten-Backend-System zur integrierten Datenanalyse in Wertschöpfungsnetzwerken zeigt. Darüber hinaus entstand, wie in Abbildung 2 ersichtlich, ein Konzept für den integrierten Referenzbaukasten inklusive des Daten-Backend-Systems. Durch die Nutzung von Personae wurden die erhobenen Anforderungen abgesichert und verallgemeinert, um den Bedarf von KMU widerzuspiegeln. Recherchen zeigen, dass bereits auf dem Markt verfügbare Softwarelösungen einen Teil der Anforderungen erfüllen, allerdings existiert keine Softwarelösung, die dies vollumfänglich tut.

\section{Ausblick und Danksagung}

Aufbauend auf den erhobenen Anforderungen werden nun einzelne Aspekte des DatenBackend-Systems in einem agilen Entwicklungsprozess ausgestaltet und umgesetzt sowie kontinuierlich mit Partnern aus dem Projekt AKKORD evaluiert. Methodisch wird zuerst ein detaillierter Abgleich der notwendigen Daten für Analyseszenarien mit den Datenquellen im Unternehmen durchgeführt. Basierend darauf wird ein initiales Datenmodell für das DatenBackend-System entwickelt, welches kompatibel zu den Datenquellen der beteiligten Unternehmen ist. Durch den Einsatz in verschiedenen Szenarien aus unterschiedlichen Bereichen des Produktlebenszyklus soll sichergestellt werden, dass die notwendige Flexibilität 
gegeben ist. Im Vordergrund stehen dabei vor allem die Integration der unterschiedlichen heterogenen Datenquellen, das Mapping der einzelnen Daten sowie die Schnittstelle zur Datenanalysesoftware.

Das Projekt AKKORD - „Vernetzte und integrierte Anwendung industrieller Datenanalyse für die wertschaffende, kompetenzorientierte Kollaboration in dynamischen Wertschöpfungsnetzwerken" wird/ wurde mit Mitteln des Bundesministeriums für Bildung und Forschung (BMBF) im Programm „Industrie 4.0 - Kollaborationen in dynamischen Wertschöpfungsnetzwerken (InKoWe)" (Förderkennzeichen 02P17D210) gefördert und vom Projektträger Karlsruhe (PTKA) betreut.

\section{Literaturverzeichnis}

[1] Göbel, Jens C. ; Eickhoff, Thomas: Konzeption von Digitalen Zwillingen smarter Produkte. In: ZWF Zeitschrift für wirtschaftlichen Fabrikbetrieb 115 (2020), special, S. 74-77

[2] Schmucker, Benedikt ; Ellinger, Johannes ; Benker, Maximilian ; Semm, Thomas ; Zäh, Michael F.: Einsatz eines Digitalen Zwillings zur Prozessoptimierung und prädiktiven Instandhaltung. In: ZWF Zeitschrift für wirtschaftlichen Fabrikbetrieb 115 (2020), special, S. 78-83

[3] Schuh, G. ; Häfner, C. ; Hopmann, C. ; Rumpe, B. ; Brockmann, M. ; Wortmann, A. ; Maibaum, J. ; Dalibor, M. ; Bibow, P. ; Sapel, P. ; Kröger, M.: Effizientere Produktion mit Digitalen Schatten. In: ZWF Zeitschrift für wirtschaftlichen Fabrikbetrieb 115 (2020), special, S. 105-107

[4] Wagner, Lars: Kein Digital Twin ohne digitale Durchgängigkeit. In: ZWF Zeitschrift für wirtschaftlichen Fabrikbetrieb 115 (2020), special, S. 43-45

[5] Wagner, Raphael ; Schleich, Benjamin ; Haefner, Benjamin ; Kuhnle, Andreas ; Wartzack, Sandro ; Lanza, Gisela: Challenges and Potentials of Digital Twins and Industry 4.0 in Product Design and Production for High Performance Products. In: Procedia CIRP 84 (2019), S. 88-93

[6] VDA (Hrsg.): FAST 2025 : Future Automotive Industry Structure. 2012

[7] Gadatsch, Andreas ; Landrock, Holm: Big Data für Entscheider : Entwicklung und Umsetzung datengetriebener Geschäftsmodelle. Wiesbaden : Springer Vieweg, 2017 (Essentials)

[8] Stark, Rainer ; Neumeyer, Sebastian ; Kim, M. ; Deuse, Jochen ; Schallow, Julian: Status Quo und Handlungsempfehlungen für die digitale Produktentstehung. In: ProduktDatenJournal 21 (2014), Nr. 2, S. 1217

[9] Mazarov, Jürgen ; Wolf, Patrick ; Schallow, Julian ; Nöhring, Fabian ; Deuse, Jochen ; Richter, Ralph: Industrial Data Science in Wertschöpfungsnetzwerken. In: ZWF Zeitschrift für wirtschaftlichen Fabrikbetrieb 114 (2019), Nr. 12, S. 874-877

[10] Gilz, Torsten ; Eigner, Martin: Ansatz zur integrierten Verwendung von SysML Modellen in PLM zur Beschreibung der funktionalen Produktarchitektur. In: Maurer, Maik; Schulze, Sven-Olaf (Hrsg.): Tag des Systems Engineering: Stuttgart 6. - 8. November 2013 ; Der Weg zu den technischen Systemen von morgen. 1. Aufl. s.I. : Carl Hanser Fachbuchverlag, 2013, S. 293-302

[11] Eigner, Martin (Hrsg.); Koch, Walter (Hrsg.); Muggeo, Christian (Hrsg.): Modellbasierter Entwicklungsprozess cybertronischer Systeme : Der PLM-unterstützte Referenzentwicklungsprozess für Produkte und Produktionssysteme. Berlin : Springer Vieweg, 2017

[12] Eigner, Martin ; Dickopf, Thomas ; Apostolov, Hristo ; Schaefer, Patrick ; Faißt, Karl-Gerhard ; Keßler, Alexander: System Lifecycle Management: Initial Approach for a Sustainable Product Development Process Based on Methods of Model Based Systems Engineering, Bd. 442. In: Fukuda, Shuichi; Bernard, Alain; Gurumoorthy, Balan; Bouras, Abdelaziz (Hrsg.): Product Lifecycle Management for a Global Market. Berlin, Heidelberg : Springer Berlin Heidelberg, 2014 (IFIP Advances in Information and Communication Technology), S. 287-300

[13] Eigner, M.: Modellbasierte Virtuelle Produktentwicklung auf einer Plattform für System Lifecycle Management. In: Sendler, Ulrich (Hrsg.): Industrie 4.0 : Beherrschung der industriellen Komplexität mit SysLM. Berlin, Heidelberg : Springer Vieweg, 2013 (Xpert.press), S. 91-110

[14] Woll, Robert ; Hayka, Haygazun ; Stark, Rainer: Ontologiebasierte Datenintegration für das Modellbasierte Systems Engineering. In: Maurer, Maik; Schulze, Sven-Olaf; Abulawi, Jutta (Hrsg.): Tag des Systems Engineering : Bremen, 12. - 14. November 2014. München : Hanser, 2015, S. 33-42

[15] Abramovici, Michael: Smart Products, Bd. 59. In: Produ, The International Academy for; Laperrière, Luc; Reinhart, Gunther (Hrsg.): CIRP Encyclopedia of Production Engineering. Berlin, Heidelberg : Springer Berlin Heidelberg, 2014, S. 1-5

[16] Eigner, Martin ; August, Urban ; Schmich, Matthias: Smarte Produkte erfordern ein Umdenken bei Produktstrukturen und Prozessen : Digitalisireung, Integration, Interdisziplinarität und Föderation. 2016

[17] Tomiyama, Tetsuo ; Lutters, Eric ; Stark, Rainer ; Abramovici, Michael: Development capabilities for smart products. In: CIRP Annals 68 (2019), Nr. 2, S. 727-750 
[18] Thomas, Dickopf ; Hristo, Apostolov ; Patrick, Müller ; Göbel, Jens Christian ; Sven, Forte: A Holistic System Lifecycle Engineering Approach - Closing the Loop between System Architecture and Digital Twins. In: Procedia CIRP 84 (2019), S. 538-544

[19] Weerawarana, Sanjiva: Web services platform architecture : SOAP, WSDL, WS-policy, WS-addressing, WSBPEL, WS-reliable messaging and more. 5. printing. Upper Saddle River, N.J. : Prentice Hall/PTR, 2008

[20] Leymann, Frank: Workflow-Based Coordination and Cooperation in a Service World, Bd. 4275. In: Meersman, Robert; Tari, Zahir (Hrsg.): On the move to meaningful Internet systems 2006: CoopIS, DOA, GADA, and ODBASE : OTM Confederated International Conferences, CoopIS, DOA, GADA, and ODBASE 2006, Montpellier, France, October 29 - November 3, 2006; proceedings, Part 1. Berlin : Springer, 2006 (Lecture Notes in Computer Science, 4275), S. 2-16

[21] Eckert, Roland ; Mansel, Wolfgang ; Specht, Gunther: STEP AP233 + standard PDM = systems engineering PDM? In: 2005 IEEE International Technology Management Conference (ICE) : IEEE, 2005 - 2005, S. 1-8

[22] AP239 Consortium: ISO 10303 (STEP) AP 239 edition 3 Application Protocol For Product Life Cycle Support (PLCS). 2015

[23] Franke, Marco ; Klein, Konstantin ; Hribernik, Karl ; Lappe, Dennis ; Veigt, Marius ; Thoben, Klaus-Dieter: Semantic Web Service Wrappers as a Foundation for Interoperability in Closed-loop Product Lifecycle Management. In: Procedia CIRP 22 (2014), S. 225-230

[24] Penciuc, Diana ; Durupt, Alexandre ; Belkadi, Farouk ; Eynard, Benoît ; Rowson, Harvey: Towards a PLM Interoperability for a Collaborative Design Support System. In: Procedia CIRP 25 (2014), S. 369-376

[25] Amsden, Jim ; Berezovskyi, Andrii: OSLC Core Version 3.0. Part 1: Overview. 2019

[26] Senington, Richard ; Baumeister, Fabian ; Ng, Amos ; Oscarsson, Jan: A linked data approach for the connection of manufacturing processes with production simulation models. In: Procedia CIRP 70 (2018), S. 440-445

[27] Fahmi Bellalouna: Integrationsplattform für eine interdisziplinäre Entwicklung mechatronischer Produkte. Ruhr-Universität Bochum, Universitätsbibliothek. Doctoralthesis. 2009

[28] Lentes, J. ; Eckstein, H. ; Zimmermann, N.: A Platform to Integrate Manufacturing Engineering and Product Lifecycle Management. In: IFAC Proceedings Volumes 45 (2012), Nr. 6, S. 1071-1076

[29] Ernst, Joscha: Systemübergreifendes Änderungsmanagement. Dissertation, 2016 (Schriftenreihe VPE Band 17)

[30] Abramovici, Michael ; Göbel, Jens Christian ; Dang, Hoang Bao: Semantic data management for the development and continuous reconfiguration of smart products and systems. In: CIRP Annals 65 (2016), Nr. 1, S. 185-188

[31] Bajaj, Manas ; Backhaus, Jonathan ; Walden, Tim ; Waikar, Manoj ; Zwemer, Dirk ; Schreiber, Chris ; Issa, Ghassan ; Martin, Lockheed: Graph-Based Digital Blueprint for Model Based Engineering of Complex Systems. In: INCOSE International Symposium 27 (2017), Nr. 1, S. 151-169

[32] Sinha, Apurva K. ; Weaver, Matt ; Morgan, Stephen G.: Visualising, Analysing, and Managing the Design Factory. In: Proceedings of the ASME 12th Biennial Conference on Engineering Systems Design and Analysis - 2014 : Presented at ASME 2014 12th Biennial Conference on Engineering Systems Design and Analysis; June 25 - 27, 2014, Copenhagen, Denmark. New York, NY : ASME, 2014

[33] Leser, Ulf ; Naumann, Felix: Informationsintegration : Architekturen und Methoden zur Integration verteilter und heterogener Datenquellen. 1. Aufl. Heidelberg : dpunkt-Verl., 2007

[34] Eickhoff, Thomas ; Eiden, Andreas ; Göbel, Jens Christian ; Eigner, Martin: A Metadata Repository for Semantic Product Lifecycle Management

[35] King, Nigel ; Horrocks, Christine ; Brooks, Joanna M.: Interviews in qualitative research. 2nd Edition. Los Angeles, London, New Delhi, Singapore, Washington DC, Melbourne : SAGE, 2019 